\title{
COMPARISONS OF MORPHOMETRIC CHARACTERISTICS AND LENGTH-WEIGHT RELATIONSHIP OF EUROPEAN EEL (ANGUILLA ANGUILLA L., 1758) IN TURKISH INLAND WATERS
}

\section{Ali İlhan; Dilek İlhan ; Ramoni Olajide Hammed}

Faculty of Fisheries, Ege University, Bornova, İzmir, Turkey

\author{
Article History: \\ Received: 1 May 2020 \\ Revised: 20 June 2020 \\ Accepted: 21 June 2020 \\ Published Online: \\ 1 July 2020 \\ Keywords: \\ Anguilla anguilla \\ Inland waters \\ LWR \\ Morphometry \\ Turkey \\ *Correspondence: \\ Dilek İlhan \\ Faculty of Fisheries \\ Ege University \\ 35100, Bornova \\ İzmir, Turkey \\ E-mail: \\ dilek.ilhan@ege.edu.tr
}

\begin{abstract}
European eels are found in all European rivers draining to seas. Comparisons of some morphometric characteristics and length-weight relationship parameters of European eel "Anguilla anguilla" collected from inland waters of Turkey were performed in this work. The 108 fish samples used in the current work were taken from the museum of Faculty of Fisheries, Ege University; they were captured from different running water bodies. Morphometric features like total length, weight, head length, head height, maximum body height, eye diameter, interorbital distance, snout length, dorsal fin length, anal fin length, pectoral fin length, predorsal distance, and preanal distance of the fish were measured. In addition, head length/maximum body height, head length/snout length, head length/head height, head length/total length, eye diameter/head length, eye diameter/inter-orbital distance, eye diameter/snout length and predorsal distance/preanal distance ratios were calculated. The analysed specimens ranged in total length from 12.70 to $64.10 \mathrm{~cm}$, and in weight between 3.21 and $416.14 \mathrm{~g}$. The lengthweight relationship parameters were estimated as $\mathrm{a}=0.011$, $\mathrm{b}=3.154$; while $\mathrm{r}^{2}$ was calculated as 0.956 .
\end{abstract}

\section{INTRODUCTION}

European eels are found in all European rivers draining to Mediterranean, North and Baltic Seas and to Atlantic south to Canary Islands; naturally only in water bodies connected to sea, stocked elsewhere. It is hypothesized to spawn only in Sargasso Sea, in Western Subtropical Atlantic; spawning peaks at beginning of March, continuing until July ${ }^{[1]}$. The feeding phase lasts for 5-8 years in males and up to 12 years or more in females. Males are rarely recorded more than $200 \mathrm{~km}$ upriver. Downstream migration starts in late summer or autumn, and adults arrive at spawning sites in the following spring. Feed on a wide variety of benthic organisms ${ }^{[1]}$. Conservation status is critically endangered (subcriteria $=\mathrm{A} 2 \mathrm{bd}$ $+4 \mathrm{bd}$ ), sharply declining. Anthropogenic factors such as overexploitation, migration barriers, pollution, habitat loss, pathogens, and parasites are the prominent factors affecting the eel population in Turkey and other countries. Adults and especially glass-eels overfished in several areas. There is no evidence that the decline is related to the infection by Anguillicola crassus $^{[1]}$. European eels have been found in Aegean 
and Mediterranean seas, also in streams and lakes connected to seas in Turkey. Because of their delicious and boneless meat, they are consumed with great delight in smoked form, especially in Europe. European eel caught from Turkish waters are exported to European countries, thereby serves as an important source of foreign exchange ${ }^{[2]}$. Eel exports were subject to quota within the scope of the "Convention on the International Trade in Endangered Species of Wild Flora and Fauna (CITES)"; in this context, 2020 export quota for eel (Anguilla anguilla) was determined as $100.000 \mathrm{~kg}$. Information about monitoring of glass eel recruitment, growth, feeding and migratory life history of European eel in Turkish waters have been reported by Yalçin and Küçük $^{[3]}$, Yalçın-Özdilek et al. ${ }^{[4]}$, YalçınÖzdilek and Solak ${ }^{[5]}$ and Linn et al. ${ }^{[6]}$, respectively. Morphometric measurement of fishes and the study of statistical relationship among them are essential for taxonomic study of a species ${ }^{[7]}$. Length-weight relationship (LWR) is an important component in fish and fisheries biology and very useful for fish population dynamics and fisheries management ${ }^{[8]}$. Turkey has an important role on the whole European eel stock with the suitable climatic conditions for growing European eel in freshwaters ${ }^{[9]}$. The principal aim of the present study was to provide data on the morphometric characteristics and LWR parameters which are necessary for conservation programs of European eel caught from Turkish inland waters.

\section{MATERIAL AND METHODS}

The 108 fishes used in this work were taken from collections of Ege University, Faculty of Fisheries Freshwater Fish Museum (ESFM/PISI). The origin of the samples (Figure 1) are Antalya Basin (Mediterranean region, ESFMPISI-1976/005, 01.05.1976, 6 specimens; Alara Stream, Alanya, ESFM-PISI1976/052, 07.08.1976, 8 specimens; Ilica Stream, Manavgat, ESFM-PISI-1992/013, 26.04.1992, 6 specimens; Karg1 Çay1, Alanya, ESFM-PISI-1976/053, 07.08.1976,
3 specimens), Western Mediterranean Basin (Eşen Stream, Kemer-Fethiye, ESFM-PISI1976/025, 17.05.1976, one specimen; Karg1 Stream, Fethiye, ESFM-PISI-1994/019, 02.05.1994, one specimen; Nasifdede Stream, Köyceğiz, ESFM-PISI-2001/131, 23.08.2001, 4 specimens; ESFM-PISI2001/154, 25.10.2001, 6 specimens; ESFMPISI-2002/005, 27.03.2002, one specimen; Yuvarlakçay Stream, Köyceğiz, ESFM-PISI2001/138, 23.08.2001, one specimen; ESFM-PISI-2001/161, 25.10.2001, 4 specimens; ESFM-PISI-2002/013, 27.03.2002, one specimen), Küçük Menderes Basin (Gümüldür brook, İzmir, ESFM-PISI1971/021, 13.06.1971, 15 specimens; ESFM-PISI-1972/020, 28.06.1972, 9 specimens; Ayranc1 brook, İzmir, ESFMPISI-1971/026, 20.06.1971, one specimen; Oğlananası pond, İzmir, ESFM-PISI1971/032, 26.06.1971, 2 specimens; Kocaçay brook, Seferihisar, ESFM-PISI1971/062, 31.07.1971, 6 specimens), Gediz Basin (old estuarin of Gediz River, ESFMPISI-1961/007, 23.10.1961, one specimen; Nif Stream, Çambel bridge, ESFM-PISI1971/036, 28.06.1971, 3 specimens; ESFMPISI-1973/001, 9.05.1973, one specimen; Göldeğirmeni spring, Tilkiköy-Manisa, ESFM-PISI-1972/007, 07.05.1972, one specimen; Ayvacik brook, Emiralem, ESFM-PISI-2001/015, 18.04.2001, one specimen), North Aegean Basin (Güzelhisar Stream, ESFM-PISI-1991/013, 27.05.1991, 3 specimens; ESFM-PISI1991/017, 28.06.1991, one specimen; Mihlıçay brook, Ayvacık, ESFM-PISI2001/079, 17.05.2001, 3 specimens; Zeytinli brook, Edremit, ESFM-PISI2001/097, 09.07.2001, one specimen; Manastır brook, Edremit, ESFM-PISI2001/101, 09.07.2001, 5 specimens; Kazan brook, Edremit, ESFM-PISI-2001/103, 10.07.2001, 3 specimens; Şahin brook, Edremit, ESFM-PISI-2001/105, 10.07.2001, 5 specimens), and Meriç-Ergene Basin (Kavak brook, Gelibolu, ESFM-PISI1982/004, 01.06.1982, one specimen; Gala Lake, ESFM-PISI-1982/017, 2.06.1982, one specimen; ESFM-PISI-1983/015, 
20.07.1983, one specimen; Baba brook, Kadıköy-Keşan, ESFM-PISI1983/004, 19.07.1983, 2 specimens). The fish samples were stored in $4 \%$ formalin solution and analysed without being sexed. Individual length was measured using $1.0 \mathrm{~mm}$ sensitive measurement ruler; while the individual weight was measured using $0.01 \mathrm{~g}$ sensitive digital scale; features like head length, head height, maximum body height, eye diameter, inter-orbital distance, snout length, dorsal fin length, anal fin length, pectoral fin length, pre-dorsal distance and preanal distance were measured with $0.01 \mathrm{~mm}$ sensitive digital compass. The pectoral fins were counted under " $\times 3$ " magnification. The equation $\mathrm{W}=\mathrm{aL}^{\mathrm{b}}$ was applied in order to establish the LWR, where $\mathrm{W}$ is the total weight $(\mathrm{g}), \mathrm{L}$ is the total length $(\mathrm{cm})$, "a" is a coefficient related to body form, and " $b$ " is an exponent indicating growth type ${ }^{[10]}$. The hypothesis of isometric growth was tested by Student's $t$-test as follows: $t s=b-3 / \operatorname{se}(b)^{[11]}$. Where " $b$ " is the slope or the growth coefficient and "se(b)" is the standard error of the slope. To know whether the fish growth is isometric or allometric, the calculated $t$-test value was compared with table critical value. Calculations were done using "Microsoft Office Professional Plus 2016" excel program.

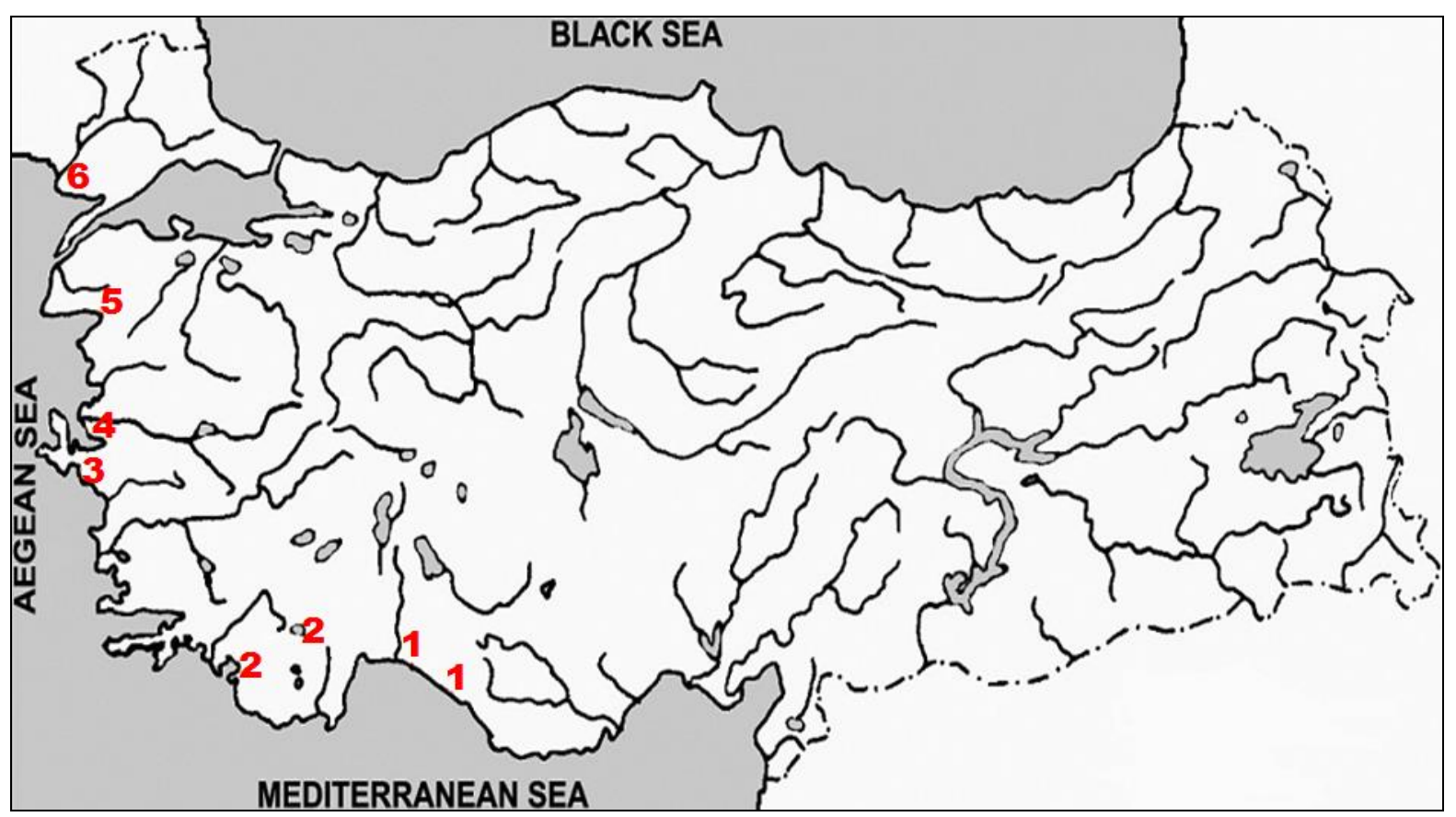

Figure 1: Map of the sampling sites. 1: Antalya Basin, 2: Western Mediterranean Basin, 3: Küçük Menderes Basin, 4: Gediz Basin, 5: North Aegean Basin, 6: Meriç-Ergene Basin.

\section{RESULTS}

Morphometric characteristics of 108 European eel fish from the six river basin of Turkish water were measured, as well as minimum, maximum and mean values were calculated. For all specimens, while total length values ranged from $12.7 \mathrm{~cm}$ to $64.1 \mathrm{~cm}$, Gediz and Western Mediterranean basins have been identified as the maximum and minimum values with the average total length of $49.40 \mathrm{~cm}$ and $23.42 \mathrm{~cm}$, respectively (Table 1). For the whole basins, the inter-orbital distance was found as $\sim 2$ times of the eye diameter. Eye diameter was found 0.40 of snout length, snout length 0.19 of head length, and head length 0.13 of total length (Table 2). Also, the mean of maximum body height was bigger than mean of head height for the specimens of all basins. Although, head height is higher than the maximum body height in some individuals, considering the mean values it 
was generally understood to be smaller (Table 2). Comparing statistics in terms of characters that can be seen; there is no difference between the basins (One-Way Analysis of Variance, ANOVA, $P>0.05$ ). The LWR parameters were given in the
Table "3". According to Student's $t$-test, we observed isometric growth in Antalya and Gediz and also positive allometric growth in Meriç-Ergene, North Aegean, Küçük Menderes, and Western Mediterranean basin for this species (Table 3).

Table 1: The morphometric characteristics of Anguilla anguilla from Turkish waters. Minimum-maximum and within the parentheses the mean \pm standard deviation.

\begin{tabular}{|c|c|c|c|c|c|c|}
\hline & $\begin{array}{l}\text { Antalya } \\
(n=23)\end{array}$ & $\begin{array}{c}\text { Western } \\
\text { Mediterranean } \\
(\mathrm{n}=19)\end{array}$ & $\begin{array}{l}\text { Gediz } \\
(n=7)\end{array}$ & $\begin{array}{l}\text { Küçük } \\
\text { Menderes } \\
(n=33)\end{array}$ & $\begin{array}{l}\text { North Aegean } \\
\quad(n=21)\end{array}$ & $\begin{array}{l}\text { Meriç-Ergene } \\
\quad(\mathrm{n}=5)\end{array}$ \\
\hline $\begin{array}{l}\mathrm{TL} \\
(\mathrm{cm})\end{array}$ & $\begin{array}{c}14.10-43.20 \\
(29.08 \pm 7.83)\end{array}$ & $\begin{array}{c}12.70-46.30 \\
(23.42 \pm 8.79)\end{array}$ & $\begin{array}{c}37.70-64.10 \\
(49.40 \pm 8.33)\end{array}$ & $\begin{array}{c}15.30-38.50 \\
(28.91 \pm 5.77)\end{array}$ & $\begin{array}{c}18.20-55.50 \\
(33.67 \pm 8.64)\end{array}$ & $\begin{array}{c}16.80-43.90 \\
(28.46 \pm 11.44)\end{array}$ \\
\hline $\begin{array}{l}\mathrm{HL} \\
(\mathrm{mm})\end{array}$ & $\begin{array}{c}19.03-59.41 \\
(38.43 \pm 11.08)\end{array}$ & $\begin{array}{c}17.18-67.06 \\
(30.75 \pm 12.05)\end{array}$ & $\begin{array}{c}49.50-75.16 \\
(65.95 \pm 9.80)\end{array}$ & $\begin{array}{c}18.37-59.66 \\
(39.47 \pm 10.10)\end{array}$ & $\begin{array}{c}23.73-79.52 \\
(43.90 \pm 11.14)\end{array}$ & $\begin{array}{c}23.57-56.72 \\
(36.69 \pm 14.06)\end{array}$ \\
\hline $\begin{array}{l}\mathrm{HH} \\
(\mathrm{mm})\end{array}$ & $\begin{array}{c}6.73-28.04 \\
(16.85 \pm 5.58)\end{array}$ & $\begin{array}{c}6.48-34.68 \\
(13.26 \pm 6.81)\end{array}$ & $\begin{array}{c}18.17-35.12 \\
(27.62 \pm 5.73)\end{array}$ & $\begin{array}{c}6.94-23.80 \\
(16.16 \pm 4.85)\end{array}$ & $\begin{array}{c}9.11-40.06 \\
(19.10 \pm 6.49)\end{array}$ & $\begin{array}{c}8.90-25.29 \\
(15.29 \pm 6.81)\end{array}$ \\
\hline $\begin{array}{l}\mathrm{MBH} \\
(\mathrm{mm})\end{array}$ & $\begin{array}{c}6.97-30.32 \\
(18.84 \pm 6.35)\end{array}$ & $\begin{array}{c}7.25-33.36 \\
(14.24 \pm 7.12)\end{array}$ & $\begin{array}{c}21.96-36.75 \\
(29.50 \pm 5.51)\end{array}$ & $\begin{array}{c}8.06-27.07 \\
(17.49 \pm 4.78)\end{array}$ & $\begin{array}{c}10.51-39.49 \\
(20.95 \pm 7.37)\end{array}$ & $\begin{array}{c}8.62-26.79 \\
(16.87 \pm 7.66)\end{array}$ \\
\hline $\begin{array}{c}\mathrm{ED} \\
(\mathrm{mm})\end{array}$ & $\begin{array}{c}1.09-4.27 \\
(2.66 \pm 0.90)\end{array}$ & $\begin{array}{c}1.14-4.62 \\
(2.26 \pm 1.06)\end{array}$ & $\begin{array}{c}3.38-5.51 \\
(4.41 \pm 0.65)\end{array}$ & $\begin{array}{c}1.30-5.55 \\
(3.15 \pm 1.07)\end{array}$ & $\begin{array}{c}1.54-5.44 \\
(3.50 \pm 1.04)\end{array}$ & $\begin{array}{c}1.45-3.51 \\
(2.26 \pm 0.81)\end{array}$ \\
\hline $\begin{array}{l}\text { IOD } \\
(\mathrm{mm})\end{array}$ & $\begin{array}{c}3.15-9.32 \\
(5.25 \pm 1.58)\end{array}$ & $\begin{array}{c}2.16-11.84 \\
(4.63 \pm 2.23)\end{array}$ & $\begin{array}{c}8.42-12.27 \\
(10.35 \pm 1.40)\end{array}$ & $\begin{array}{c}3.19-8.49 \\
(5.83 \pm 1.49)\end{array}$ & $\begin{array}{c}3.61-15.60 \\
(7.31 \pm 2.93)\end{array}$ & $\begin{array}{c}3.29-7.46 \\
(4.88 \pm 1.75)\end{array}$ \\
\hline $\begin{array}{l}\mathrm{NL} \\
(\mathrm{mm})\end{array}$ & $\begin{array}{c}3.47-12.32 \\
(7.20 \pm 2.24)\end{array}$ & $\begin{array}{c}2.78-13.09 \\
(5.79 \pm 2.47)\end{array}$ & $\begin{array}{c}9.41-15.28 \\
(12.67 \pm 2.01)\end{array}$ & $\begin{array}{c}3.89-10.69 \\
(7.36 \pm 1.68)\end{array}$ & $\begin{array}{c}4.12-16.29 \\
(8.53 \pm 3.08)\end{array}$ & $\begin{array}{c}4.31-10.23 \\
(6.45 \pm 2.41)\end{array}$ \\
\hline $\begin{array}{l}\text { DFL } \\
(\mathrm{cm})\end{array}$ & $\begin{array}{c}10.30-29.80 \\
(20.64 \pm 5.43)\end{array}$ & $\begin{array}{c}8.40-31.90 \\
(16.33 \pm 6.22)\end{array}$ & $\begin{array}{c}26.10-46.90 \\
(34.70 \pm 6.42)\end{array}$ & $\begin{array}{c}11.10-25.70 \\
(20.20 \pm 3.83)\end{array}$ & $\begin{array}{c}13.20-37.50 \\
(24.00 \pm 6.09)\end{array}$ & $\begin{array}{c}12.20-32.10 \\
(20.56 \pm 8.34)\end{array}$ \\
\hline $\begin{array}{l}\mathrm{DFH} \\
(\mathrm{mm})\end{array}$ & $\begin{array}{c}1.65-7.59 \\
(4.83 \pm 1.69)\end{array}$ & $\begin{array}{c}1.09-8.26 \\
(2.67 \pm 1.73)\end{array}$ & $\begin{array}{c}6.31-9.45 \\
(7.83 \pm 1.20)\end{array}$ & $\begin{array}{c}1.04-6.63 \\
(3.96 \pm 1.52)\end{array}$ & $\begin{array}{c}2.38-11.65 \\
(5.40 \pm 2.20)\end{array}$ & $\begin{array}{c}2.08-6.19 \\
(3.88 \pm 1.72)\end{array}$ \\
\hline $\begin{array}{l}\text { AFL } \\
(\mathrm{cm})\end{array}$ & $\begin{array}{c}8.70-24.30 \\
(17.22 \pm 4.43)\end{array}$ & $\begin{array}{c}7.90-27.80 \\
(14.04 \pm 5.17)\end{array}$ & $\begin{array}{c}22.30-41.10 \\
(28.91 \pm 5.79)\end{array}$ & $\begin{array}{c}9.40-21.40 \\
(16.99 \pm 3.23)\end{array}$ & $\begin{array}{c}10.80-32.00 \\
(20.04 \pm 4.90)\end{array}$ & $\begin{array}{c}10.00-26.30 \\
(17.16 \pm 6.72)\end{array}$ \\
\hline $\begin{array}{l}\mathrm{AFH} \\
(\mathrm{mm})\end{array}$ & $\begin{array}{c}1.08-7.18 \\
(4.61 \pm 1.64)\end{array}$ & $\begin{array}{c}1.11-8.73 \\
(2.91 \pm 1.87)\end{array}$ & $\begin{array}{c}5.46-8.66 \\
(6.94 \pm 1.22)\end{array}$ & $\begin{array}{c}1.32-6.86 \\
(3.80 \pm 1.41)\end{array}$ & $\begin{array}{c}1.97-9.85 \\
(4.82 \pm 1.77)\end{array}$ & $\begin{array}{c}2.03-6.77 \\
(3.96 \pm 1.82)\end{array}$ \\
\hline $\begin{array}{l}\text { PFL } \\
(\mathrm{mm})\end{array}$ & $\begin{array}{c}2.34-7.97 \\
(5.11 \pm 1.54)\end{array}$ & $\begin{array}{c}1.87-9.62 \\
(3.96 \pm 1.84)\end{array}$ & $\begin{array}{c}3.95-10.09 \\
(7.72 \pm 1.96)\end{array}$ & $\begin{array}{c}1.29-7.34 \\
(4.85 \pm 1.48)\end{array}$ & $\begin{array}{c}3.01-10.32 \\
(5.80 \pm 1.77)\end{array}$ & $\begin{array}{c}2.72-7.53 \\
(4.84 \pm 2.15)\end{array}$ \\
\hline $\begin{array}{l}\text { PDD } \\
(\mathrm{cm})\end{array}$ & $\begin{array}{c}3.80-13.20 \\
(8.39 \pm 2.44)\end{array}$ & $\begin{array}{c}3.40-14.20 \\
(6.73 \pm 2.78)\end{array}$ & $\begin{array}{c}11.30-16.90 \\
(14.53 \pm 2.18)\end{array}$ & $\begin{array}{l}4.10-12.41 \\
(862 \pm 1.92)\end{array}$ & $\begin{array}{c}5.00-17.70 \\
(9.55 \pm 2.62)\end{array}$ & $\begin{array}{c}4.60-11.60 \\
(7.82 \pm 3.04)\end{array}$ \\
\hline $\begin{array}{l}\text { PAD } \\
(\mathrm{mm})\end{array}$ & $\begin{array}{c}5.40-18.90 \\
(11.84 \pm 3.49)\end{array}$ & $\begin{array}{c}4.80-18.50 \\
(9.38 \pm 3.62)\end{array}$ & $\begin{array}{c}15.40-23.50 \\
(20.49 \pm 3.16)\end{array}$ & $\begin{array}{c}5.90-17.10 \\
(11.94 \pm 2.60)\end{array}$ & $\begin{array}{c}7.30-23.50 \\
(13.60 \pm 3.79)\end{array}$ & $\begin{array}{c}6.80-17.60 \\
(11.30 \pm 4.73)\end{array}$ \\
\hline $\begin{array}{l}\text { TW } \\
(\mathrm{g})\end{array}$ & $\begin{array}{c}4.53-177.82 \\
(64.51 \pm 45.24)\end{array}$ & $\begin{array}{c}3.21-216.67 \\
(38.89 \pm 52.71)\end{array}$ & $\begin{array}{c}98.79-339.62 \\
(230.20 \pm 92.94)\end{array}$ & $\begin{array}{c}5.06-126.41 \\
(53.77 \pm 31.47)\end{array}$ & $\begin{array}{c}12.18-416.14 \\
(99.56 \pm 99.53)\end{array}$ & $\begin{array}{c}8.52-178.20 \\
(69.78 \pm 70.76)\end{array}$ \\
\hline
\end{tabular}

$\mathrm{n}$ : number of specimens, TL: total length, HL: head length, HH: head height, MBH: maximum body height, ED: eye diameter, IOD: inter-orbital distance, NL: snout length, DFL: dorsal fin length, DFH: dorsal fin height, AFL: anal fin length, AFH: anal fin height, PFL: pectoral fin length, PDD: predorsal distance, PAD: preanal distance, TW: total weight. 
Table 2: The ratios between morphometric characteristics of Anguilla anguilla from Turkish waters. Minimum-maximum and within the parentheses the mean \pm standard deviation.

\begin{tabular}{lccccccc}
\hline & $\begin{array}{c}\text { Antalya } \\
(\mathrm{n}=23)\end{array}$ & $\begin{array}{c}\text { Western } \\
\text { Mediterranean } \\
(\mathrm{n}=19)\end{array}$ & $\begin{array}{c}\text { Gediz } \\
(\mathrm{n}=7)\end{array}$ & $\begin{array}{c}\text { Küçük } \\
\text { Menderes } \\
(\mathrm{n}=33)\end{array}$ & $\begin{array}{c}\text { North } \\
\text { Aegean } \\
(\mathrm{n}=21)\end{array}$ & $\begin{array}{c}\text { Meriç- } \\
\text { Ergene } \\
(\mathrm{n}=5)\end{array}$ & ANOVA \\
\cline { 2 - 8 } ED/HL & $0.05-0.10$ & $0.05-0.12$ & $0.06-0.08$ & $0.05-0.11$ & $0.06-0.13$ & $0.06-0.08$ & $(\mathrm{~F}=2.24$, \\
& $(0.08 \pm 0.01)$ & $(0.08 \pm 0.02)$ & $(0.07 \pm 0.01)$ & $(0.08 \pm 0.01)$ & $(0.08 \pm 0.02)$ & $(0.07 \pm 0.01)$ & $P>0.05)$ \\
ED/IOD & $0.41-0.62$ & $0.28-0.78$ & $0.35-0.71$ & $0.34-0.67$ & $0.18-0.73$ & $0.45-0.52$ & $(\mathrm{~F}=2.20$, \\
& $(0.52 \pm 0.07)$ & $(0.52 \pm 0.14)$ & $(0.55 \pm 0.11)$ & $(0.51 \pm 0.08)$ & $(0.51 \pm 0.12)$ & $(0.49 \pm 0.03)$ & $P>0.05)$ \\
ED/NL & $0.31-0.50$ & $0.28-0.68$ & $0.31-0.46$ & $0.31-0.57$ & $0.17-0.70$ & $0.34-0.45$ & $(\mathrm{~F}=1.67$, \\
& $(0.40 \pm 0.06)$ & $(0.42 \pm 0.12)$ & $(0.37 \pm 0.06)$ & $(0.42 \pm 0.08)$ & $(0.43 \pm 0.12)$ & $(0.38 \pm 0.04)$ & $P>0.05)$ \\
HH/MBH & $0.73-1.16$ & $0.62-1.09$ & $0.85-1.00$ & $0.66-1.11$ & $0.74-1.08$ & $0.85-1.03$ & $(\mathrm{~F}=0.38$, \\
& $(0.91 \pm 0.12)$ & $(0.91 \pm 0.12)$ & $(0.91 \pm 0.05)$ & $(0.92 \pm 0.09)$ & $(0.91 \pm 0.09)$ & $(0.95 \pm 0.06)$ & $P>0.05)$ \\
NL/HL & $0.17-0.22$ & $0.16-0.24$ & $0.17-0.19$ & $0.15-0.20$ & $0.16-0.42$ & $0.18-0.19$ & $(\mathrm{~F}=0.42$, \\
& $(0.19 \pm 0.01)$ & $(0.19 \pm 0.02)$ & $(0.18 \pm 0.01)$ & $(0.18 \pm 0.01)$ & $(0.20 \pm 0.05)$ & $(0.18 \pm 0.01)$ & $P>0.05)$ \\
HH/HL & $0.32-0.44$ & $0.31-0.50$ & $0.39-0.46$ & $0.35-0.52$ & $0.36-0.59$ & $0.38-0.45$ & $(\mathrm{~F}=1.03$, \\
& $(0.40 \pm 0.03)$ & $(0.40 \pm 0.05)$ & $(0.42 \pm 0.02)$ & $(0.41 \pm 0.04)$ & $(0.44 \pm 0.05)$ & $(0.41 \pm 0.02)$ & $P>0.05)$ \\
HL/TL & $0.11-0.15$ & $0.11-0.16$ & $0.12-0.15$ & $0.12-0.24$ & $0.10-0.14$ & $0.12-0.14$ & $(\mathrm{~F}=0.57$, \\
& $(0.13 \pm 0.10)$ & $(0.13 \pm 0.01)$ & $(0.14 \pm 0.01)$ & $(0.14 \pm 0.02)$ & $(0.13 \pm 0.01)$ & $(0.13 \pm 0.01)$ & $P>0.05)$ \\
PDD/PAD & $0.69-0.78$ & $0.67-0.96$ & $0.67-0.75$ & $0.65-0.77$ & $0.62-0.77$ & $0.66-0.72$ & $(\mathrm{~F}=0.96$, \\
& $(0.73 \pm 0.02)$ & $(0.72 \pm 0.06)$ & $(0.70 \pm 0.03)$ & $(0.71 \pm 0.03)$ & $(0.70 \pm 0.04)$ & $(0.69 \pm 0.02)$ & $P>0.05)$ \\
\hline
\end{tabular}

$\mathrm{n}$ : number of specimens, TL: total length, HL: head length, HH: head height, MBH: maximum body height, ED: eye diameter, IOD: inter-orbital distance, NL: snout length, PDD: predorsal distance, PAD: preanal distance.

Table 3: Length-weight relationship parameters of Anguilla anguilla from Turkish waters.

\begin{tabular}{ccccccc} 
& $\begin{array}{c}\text { Antalya } \\
(\mathrm{n}=23)\end{array}$ & $\begin{array}{c}\text { Western } \\
\text { Mediterranean } \\
(\mathrm{n}=19)\end{array}$ & $\begin{array}{c}\text { Gediz } \\
(\mathrm{n}=7)\end{array}$ & $\begin{array}{c}\text { Küçük } \\
\text { Menderes } \\
(\mathrm{n}=33)\end{array}$ & $\begin{array}{c}\text { North } \\
\text { Aegean } \\
(\mathrm{n}=21)\end{array}$ & $\begin{array}{c}\text { Meriç- } \\
\text { Ergene } \\
(\mathrm{n}=5)\end{array}$ \\
\cline { 2 - 7 } & 0.0043 & 0.0004 & 0.0084 & 0.0006 & 0.0007 & 0.0012 \\
$\mathrm{a}$ & 2.794 & 3.438 & 2.604 & 3.347 & 3.293 & 3.136 \\
$\mathrm{~b}$ & 0.2513 & 0.0804 & 0.4603 & 0.1089 & 0.1120 & 0.0396 \\
$\mathrm{se}(\mathrm{b})$ & 0.855 & 0.991 & 0.865 & 0.968 & 0.979 & 0.999 \\
$\mathrm{r}{ }^{2}$ & -0.8189 & 5.4393 & -0.8606 & 3.1850 & 2.6207 & 3.4422 \\
$t$-test & $\mathrm{I}$ & $\mathrm{A}+$ & $\mathrm{I}$ & $\mathrm{A}+$ & $\mathrm{A}+$ & $\mathrm{A}+$ \\
$\mathrm{GT}$ & & & & & &
\end{tabular}

$\mathrm{n}$ : number of specimens, a: intercept, b: slope, se(b): standard error of the slope, $\mathrm{r}^{2}$ : coefficient of determination, GT: growth type, A+: positive allometric, I: Isometric.

\section{DISCUSSION}

The examined 108 European eel in our study showed total length between 12.7 and $64.1 \mathrm{~cm}$. Other works on European eel of Turkish water stated individual length varying between 6.5 and $92.0 \mathrm{~cm}^{[4]}$. However, works on European eel of European water reported individual lengths to vary between 5.0 and $121.5 \mathrm{~cm}$ (Table 4) ${ }^{[12-19]}$. In the present study, the LWR parameters were found as $\mathrm{a}=0.004-0.0084, \quad \mathrm{~b}=2.604-3.438, \quad \mathrm{r}^{2}=0.855-$ 0.999. The fish species was found to show positive allometric growth type in Meriç-Ergene, North Aegean, Küçük Menderes, and Western Mediterranean basin, and isometric growth in Antalya and Gediz. Works done on Turkish and European waters reported that "a" value varies between 0.0003 and 0.0106 , "b" value varies between 2.596 and 3.470 , and $\mathrm{r}^{2}$ value varies between 0.652 and 0.999 
Table 4: Length-weight relationship parameters of Anguilla anguilla from different localities.

\begin{tabular}{|c|c|c|c|c|c|c|c|c|}
\hline Locality & $\mathrm{n}$ & $\mathrm{TL}(\mathrm{cm})$ & $\mathrm{a}$ & $\mathrm{b}$ & se(b) & $r^{2}$ & GT & Author \\
\hline $\begin{array}{l}\text { Averio Lagoon } \\
\text { (Portugal) }\end{array}$ & 1170 & $5.0-59.0$ & 0.0006 & 3.281 & - & - & - & Gordo and Jorge $^{[9]}$ \\
\hline $\begin{array}{l}\text { Rihios Estuary } \\
\text { (Grecee) }\end{array}$ & 7 & $5.7-49.5$ & 0.0003 & 3.470 & - & 0.999 & - & $\begin{array}{l}\text { Koutrakis and } \\
\text { Tsikliras }^{[10]}\end{array}$ \\
\hline $\begin{array}{l}\text { Asi River } \\
\text { (Turkey) }\end{array}$ & 315 & $6.5-92.0$ & 0.0007 & 3.270 & - & 0.960 & - & $\begin{array}{l}\text { Yalçın-Özdilek } \\
\text { et al. } .^{[4]}\end{array}$ \\
\hline $\begin{array}{l}\text { Hatay Region } \\
\text { (Turkey) }\end{array}$ & 212 & $45.1-61.8$ & 0.0050 & 2.767 & - & 0.844 & - & Özcan ${ }^{[11]}$ \\
\hline $\begin{array}{l}\text { Arade Estuary } \\
\text { (Southern Portugal) }\end{array}$ & 107 & $17-68.6$ & 0.0010 & 3.160 & - & 0.985 & $\mathrm{~A}+$ & Veiga et al. ${ }^{[12]}$ \\
\hline $\begin{array}{l}\text { Flanders } \\
\text { (Belgium) }\end{array}$ & 17586 & $6.8-121.5$ & 0.0011 & 3.130 & - & 0.980 & - & $\begin{array}{l}\text { Verreycken } \\
\text { et al. }{ }^{[13]}\end{array}$ \\
\hline $\begin{array}{l}\text { Donana Marshlands } \\
\text { (Southwest Spain) }\end{array}$ & 512 & $5.3-67.0$ & 0.0010 & 3.280 & - & 0.991 & - & $\begin{array}{l}\text { Moreno-Valcárcel } \\
\text { et al. } .^{[14]}\end{array}$ \\
\hline $\begin{array}{l}\text { Cetina River } \\
\text { (Southern Croatia) }\end{array}$ & 36 & $24.0-42.0$ & 0.0081 & 2.664 & - & 0.652 & - & Piria et al..$^{[15]}$ \\
\hline $\begin{array}{l}\text { Main and Lateral } \\
\text { Channels (Croatia) }\end{array}$ & 21 & $18.0-61.5$ & 0.0050 & 2.816 & - & 0.849 & - & Piria et al..$^{[15]}$ \\
\hline $\begin{array}{l}\text { Jadro River } \\
\text { (Croatia) }\end{array}$ & 151 & $18.0-70.0$ & 0.0066 & 2.724 & - & 0.911 & - & Piria et al. ${ }^{[15]}$ \\
\hline $\begin{array}{l}\text { Žrnovnica river } \\
\text { (Croatia) }\end{array}$ & 171 & $13.0-63.0$ & 0.0106 & 2.596 & - & 0.899 & - & Piria et al. ${ }^{[15]}$ \\
\hline $\begin{array}{l}\text { Ljuta River } \\
\text { (Croatia) }\end{array}$ & 1 & 76.0 & - & - & - & - & - & Piria et al. ${ }^{[15]}$ \\
\hline $\begin{array}{l}\text { Gediz Estuary in İzmir } \\
\text { Bay (Turkey) }\end{array}$ & 73 & $32.8-59.7$ & 0.0005 & 3.270 & - & 0.9935 & - & Kara et al..$^{[16]}$ \\
\hline $\begin{array}{l}\text { Antalya basin } \\
\text { (Turkey) }\end{array}$ & 23 & $14.1-43.2$ & 0.0043 & 2.794 & 0.251 & 0.855 & I & This study \\
\hline $\begin{array}{l}\text { Western Mediterranean } \\
\text { Basin (Turkey) }\end{array}$ & 19 & $12.7-46.3$ & 0.0004 & 3.438 & 0.080 & 0.991 & $\mathrm{~A}+$ & This study \\
\hline Gediz Basin (Turkey) & 7 & $37.7-64.1$ & 0.0084 & 2.604 & 0.460 & 0.865 & I & This study \\
\hline $\begin{array}{l}\text { Küçük Menderes Basin } \\
\text { (Turkey) }\end{array}$ & 33 & $15.3-38.5$ & 0.0006 & 3.347 & 0.109 & 0.968 & $\mathrm{~A}+$ & This study \\
\hline $\begin{array}{l}\text { North Aegean Basin } \\
\text { (Turkey) }\end{array}$ & 21 & $18.2-55.5$ & 0.0007 & 3.293 & 0.112 & 0.979 & $\mathrm{~A}+$ & This study \\
\hline $\begin{array}{l}\text { Meriç-Ergene Basin } \\
\text { (Turkey) }\end{array}$ & 5 & $16.8-43.9$ & 0.0012 & 3.136 & 0.040 & 0.999 & $\mathrm{~A}+$ & This study \\
\hline
\end{tabular}

$\mathrm{n}$ : number of specimens, TL: total length, a: intercept, b: slope, se(b): standard error of the slope, $\mathrm{r}^{2}$ : coefficient of determination, GT: growth type, A+: positive allometric, I: Isometric.

$(\text { Table } 4)^{[12-19]}$. As for growth type, only the work in South Portugal reported positive allometric growth for this species
(Table 4$)^{[15]}$. The LWR in fishes can be affected by several factors including habitat, area, season, degree of stomach fullness, 
gonad maturity, sex, health, preservation techniques, and differences in the observed length ranges of the specimen caught ${ }^{[20]}$, which were not accounted for in the present study.

Despite the fact that the Deepwater Horizon Explosion in year 2010, which occurred at Gulf of Mexico "the site of reproduction for European eel", was connected to oil pollution; FAO records showed that there is no big alteration in the World's sea catch quantity. However, according to TUIK (Turkish Statistical Institute) statistics ${ }^{[21]}$, data on fish catch from our coasts showed that there was a reduction in the quantity of harvested fish from 1997 (where the highest quantity of 400 tons was recorded), especially after 2010 (where around 50 tons was recorded). It could be said that constructed dams especially on streams that empty into Mediterranean and Aegean seas have high influence on our country's inland waters' decline in yield. According to the General Directorate of State Hydraulic Works (DSI) ${ }^{[22]}$ data, 595 dams were built and operated for various purposes from 1936 until today. Because of dams, the possible migration routes of eels (streams spilled in the Marmara, Aegean, and Mediterranean Sea) was $\sim 20 \%$ before 2000 , today this ratio is raised $\sim 32 \%$. Furthermore, increment in stream pollution could also be considered as another factor.

\section{FUNDING SOURCE DISCLOSURE}

This research received no specific grant from any funding agency in the public, commercial, or not-for-profit sectors.

\section{CONFLICT OF INTEREST}

The authors have no potential financial conflict of interest.

\section{REFERENCES}

[1] Kottelat, M. and Freyhof, J. (2007). Handbook of European Freshwater Fishes. Publications Kottelat, Cornol, Switzerland.
[2] Geldiay, R. and Balık, S. (2007). Freshwater Fishes of Turkey, V. Edition. Ege University Press, Bornova, İzmir, Turkey.

[3] Yalçın, Ş. and Küçük, F. (2002). Monitoring of glass eel recruitment in Turkey. In: Monitoring of Glass Eel Recruitment (Dekker, W., ed.), pp. 241-256. Netherlands Institute of Fisheries Research, IJmuiden, Netherlands.

[4] Yalçın-Özdilek, Ş.; Gümüş, A. and Dekker, W. (2006). Growth of European eel in a Turkish River at the Southern-Eastern limit of its distribution. Electronic Journal of Ichthyology, 2006(2): 55-64.

[5] Yalçın-Özdilek, Ş. and Solak, K. (2007). The Feeding of European eel, Anguilla anguilla L. in the River Asi, Turkey. Electronic Journal of Ichthyology, 2007(1): 26-34.

[6] Lin, Y.-J.; Yalçın-Özdilek, Ş.; Lizuka, Y. et al. (2011). Migratory life history of European eel Anguilla anguilla from freshwater regions of the River Asi, southern Turkey and their high otolith $\mathrm{Sr}: \mathrm{Ca}$ ratios. Journal of Fish Biology, 78(3): 860-868.

[7] Tandon K. K.; Johal M. S. and Bala, S. (1993). Morphometry of Cirrhinus reba (Hamilton) from Kanjli wetland, Punjab, India. Res Bull Punjab Univ Sci, 43(1-4): 73-78.

[8] Froese, R.; Tsikliras, A. C. and Stergiou, K. I. (2011): Editorial note on weight-length relations of fishes. Acta Ichthyologica et Piscatoria 41(4), 261-263.

[9] Yalçın-Özdilek, Ş. (2016). The European eel in Turkey. In: The Turkish Part of the Mediterranean Sea. Marine Biodiversity, Fisheries, Conservation and Governance (Turan, C.; Salihoğlu, B.; Özgür-Özbek, E. et al., eds), pp. 407-424. Turkish Marine Research Foundation (TUDAV), İstanbul, Turkey.

[10] Ricker, W. E. (1975). Computation and Interpretation of Biological 
Statistics of Fish Populations. Bulletin of the Fisheries Research Board of Canada, Bulletin 191. Department of the Environment Fisheries and Marine Service, Ottawa, Canada.

[11] Sokal, R. R. and Rohlf, J. F. (1987). Introduction to Biostatistics. WH Freeman and Co, New York, NY, USA.

[12] Gordo, L. S. and Jorge, I. M. (1991). Age and growth of the Europen eel, Anguilla anguilla (Linnaeus, 1758), in the Averio lagoon, Portugal. Scientia Marina, 55(2): 389-395.

[13] Koutrakis, E. T. and Tsikliras, A. C. (2003). Length-weight relationships of fishes from three northern Aegean estuarine systems (Greece). Journal of Applied Ichthyology, 19(4): 258-260.

[14] Özcan, G. (2008). Length-weight relationships of five freshwater fish species from the Hatay province, Turkey. Journal of FisheriesSciences.com, 2(1):51-53.

[15] Veiga, P.; Machado, D.; Almeida, C. et al. (2009). Weight-length relationships for 54 species of the Arade estuary, southern Portugal. Journal of Applied Ichthyology, 25(4): 493-496.

[16] Verreycken, H.; Van Thuyne, G. and Belpaire, C. (2011). Lengthweight relationships of 40 freshwater fish species from two decades of monitoring in Flanders (Belgium). Journal of Applied Ichthyology, 27(6): 1416-1421.

[17] Moreno-Valcárcel, R.; Oliva-Paterna, F. J.; Arribas, C. et al. (2012). Lengthweight relationships for 13 fish species collected in the Doñana marshlands (Guadalquivir estuary, SW Spain). Journal of Applied Ichthyology 28(4): 663-664.

[18] Piria, M.; Šprem, N.; Tomljanović, T. et al. (2014). Length-weight relationships of European eel Anguilla anguilla (Linnaeus, 1758) from six karst catchments of the Adriatic basin, Croatia. Croatian Journal of Fisheries, 72: 32-35.

[19] Kara, A.; Sağlam, C.; Acarlı, D. and Cengiz, Ö. (2017). Length-weight relationships for 48 fish species of the Gediz estuary, in İzmir Bay (Central Aegean Sea, Turkey). Journal of the Marine Biological Association of the United Kingdom, 98 (4): 879-884.

[20] Tesch, F. W. (1971). Age and growth. In: Methods for Assessment of Fish Production in Fresh Waters (Ricker, W. E. ed.), pp. 99-130. Blackwell Scientific Publications, Oxford.

[21] TUİK (2020). Turkish Statistical Institute. Databases, Fishery Statistics (http://www.turkstat.gov.tr).

[22] DSI. Search Dam - General Directorate of State Hydraulic Works (http://en.dsi.gov.tr/search-dam).

\section{How to cite this article:}

İlhan, A.; İlhan, D. and Hammed, R. O. (2020). Comparisons of morphometric characteristics and length-weight relationship of European eel (Anguilla anguilla L., 1758) in Turkish inland waters. Egyptian Journal of Zoology, 74: 13-21 (DOI: 10.12816/ejz.2020.29177.1030). 


\title{
مقارنات للخصائص المورفومترية وعلاقة الطول والوزن لثعبان البحر الأوروبي

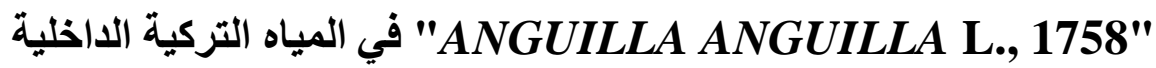

\author{
علي إلهان، ديليك إلهان، راموني أولاجيد حامد
}

كلية الثروة السمكية، جامعة إيجه، بورنوفا، إزمير، تركيا

توجد ثعابين البحر الأوروبية في جميع الأنهار الأوروبية التي تصب في البحار. وقد أجريت في هذه الدراسة مقارنات

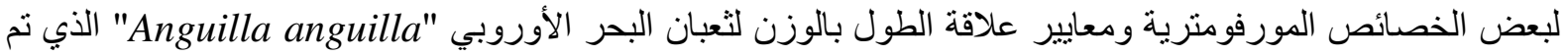

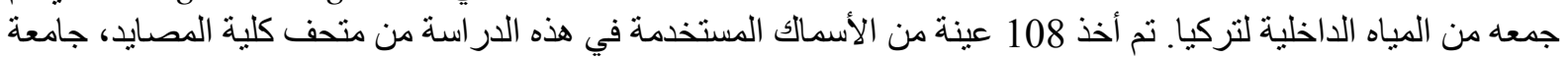

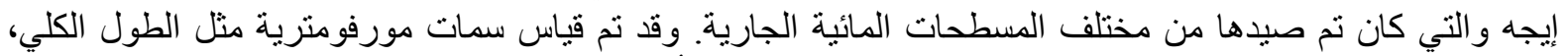

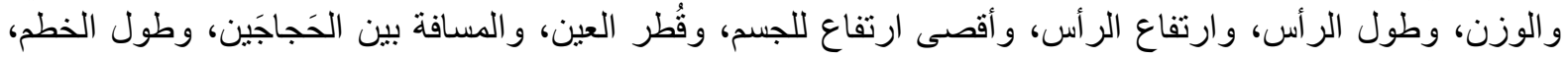

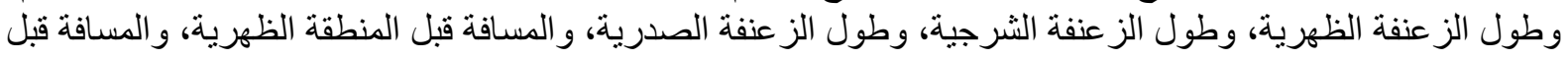

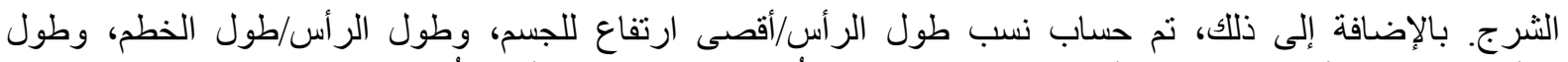

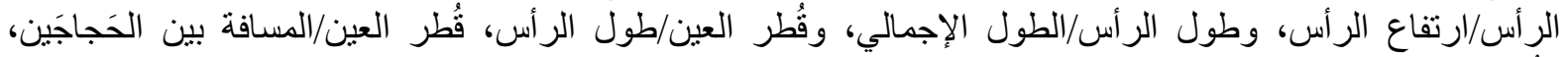

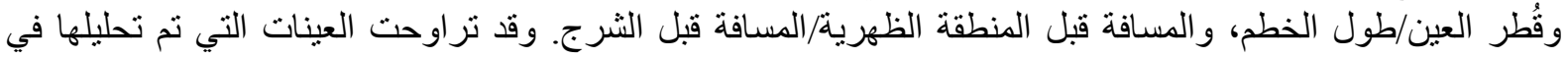

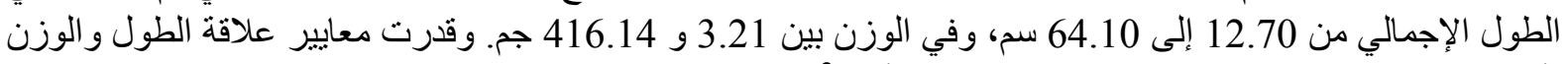

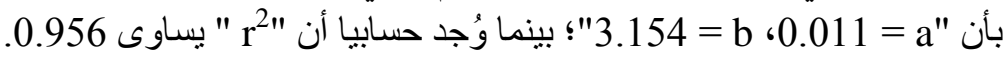

